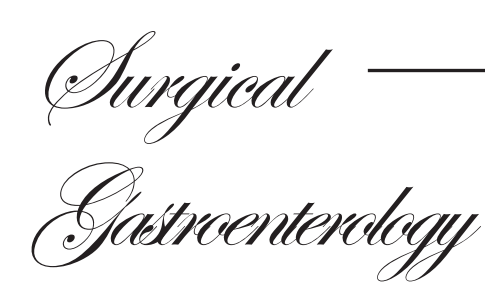

\title{
Community acquired secondary bacterial peritonitis in a tertiary hospital of South India: an audit with special reference to peritoneal fluid culture
}

\author{
Vishnu Prasad Nelamangala Ramakrishnaiah, ${ }^{1}$ Chandramaliteswaran \\ Chandrakasan, ${ }^{1}$ Kadambari Dharanipragadha, ${ }^{1}$ Sujatha Sistla, ${ }^{2}$ \\ Srinivasan Krishnamachari ${ }^{1}$
}

\section{ABSTRACT}

Departments of Surgery ${ }^{1}$ and Microbiology ${ }^{2}$

Jawaharlal Institute of Postgraduate Medical Education and Research (JIPMER),

Puducherry - 605006, India

\section{Correspondence:}

Dr. Vishnu Prasad N. R.

Email: vprasad285@gmail.com
Background: This study was conducted to elucidate the spectrum of community acquired acute bacterial peritonitis, the role of microbiological culture in its management and other factors affecting its outcome.

Methods: This was a prospective study wherein we examined cases of secondary bacterial peritonitis admitted and operated at our institution from January 2005 to May 2006. The peritoneal fluid was sent for bacterial culture and sensitivity testing. Patients were followed up with relevant progress details till discharge or death.

Results: We enrolled 352 patients. The mean age of the study population was 42.4 years with a male:female ratio of 7:1. Gastroduodenal perforations formed the major site of perforation $(51 \%)$, followed by small bowel $(29 \%)$ and appendicular perforations (17\%). Culture positivity rate was $64 \%$. Escherichia coli and Klebsiella species were the predominant isolates from peritoneal fluid. These main isolates were predominantly sensitive to ceftazidime, amikacin and chloramphenicol. Ampicillin with gentamicin and metronidazole was the first line of treatment used preoperatively in $67 \%$ of the patients, given its low cost and easier availability. The overall morbidity and mortality rates were $52 \%$ and $16.5 \%$ respectively. $78 \%$ of patients received inadequate antibiotics preoperatively. Only $26 \%$ had appropriate change of antibiotics postoperatively.

Conclusions: There was no significant benefit of postoperative change of antibiotics based on culture results. Analysis of factors influencing mortality shows dominance of host related factors over the type and source of infection with high risk population identified by age $>60$ years, delayed presentations $>3$ days and APACHE II score $>15$.

KEYWORDS: peritonitis, perforation, management, prognosis

\section{Introduction}

Secondary bacterial peritonitis is common in South India and its spectrum differs from the West. Many of these patients present late. Information obtained by peritoneal fluid culture is valuable for management of future patients. ${ }^{1-3}$ Such data is scarce from South India. The present study was planned to study the spectrum of secondary peritonitis pathogens, factors affecting the outcome and the role of microbiological culture in their management. 


\section{Methods}

This prospective study of community acquired generalized secondary bacterial peritonitis included all cases of secondary bacterial peritonitis treated by our Department of Surgery from January 2005 to May 2006. Localized peritonitis, pelvic peritonitis, pediatric patients, peritonitis following dialysis or elective surgery and patients who expired before surgery, were excluded. Baseline clinical data including age, gender, duration of acute symptoms, major medical illness, vital signs such as hypotension, tachycardia, tachypnea and clinical examination findings were recorded at admission. Chest radiograph was done in all cases and ultrasonogram in selected cases. Biochemical parameters like serum electrolytes, urea and creatinine were estimated in all cases. APACHE II score was calculated after arterial blood gas analysis.

Preoperative organ impairment was defined by following criteria:

I. Cardiovascular: heart rate $>110$ per minute after adequate resuscitation or requirement of inotropic support or electrocardiographic evidence of ischemia or infarct.

II. Hypotension: systolic blood pressure $<100 \mathrm{mmHg}$.

III. Pulmonary impairment: clinical evidence of respiratory distress supported by chest X-ray findings or history of treatment for chronic obstructive pulmonary disease or pulmonary tuberculosis with reduction in exercise tolerance or $\mathrm{PaO}_{2}<65 \mathrm{mmHg}$ or patient requiring mechanical ventilation.

IV. Renal failure: serum creatinine $>2.0 \mathrm{mg} / \mathrm{dl}$ and serum urea $>40 \mathrm{mg} / \mathrm{dl}$ with or without oliguria after adequate fluid resuscitation.

V. Liver disease: documented cirrhosis or a serum bilurubin level $>2.0 \mathrm{mg} / \mathrm{dl}$ or previous episode of hepatic failure or encephalopathy.

VI. Diabetes mellitus: patient requiring insulin or oral hypoglycemic agents.

After resuscitation patients were taken up for exploratory laparotomy. The delay from time of diagnosis to surgery was noted. At laparotomy, intraoperative findings were noted in relation to site of origin, quantity and nature of peritoneal fluid and the surgical procedure carried out. The peritoneal fluid was sent for bacterial culture and sensitivity by standard methods and reported after 72 hours of incubation. Anaerobic culture was attempted whenever facility was available. Routine in-vitro antibiotic sensitivity testing was done with appropriate panel of antibiotics as per standard methods. Preoperative antibiotics were defined as antibiotics given at the time of admission prior to surgery, and immediate postoperative antibiotics were those given following surgery and before the availability of microbiology reports. Patients were followed up noting relevant progress details including postoperative hemoglobin level, culture results, postoperative change of antibiotics, ICU stay, repeat laparotomy, wound infection and other morbidities till discharge or death.

Preoperative antibiotic therapy was considered adequate if the administered antibiotics covered all the organisms isolated while the rest were considered inadequate. Postoperative antibiotic change was considered appropriate if any of the changed antibiotics correlated with antibiogram results, even if the change was made without consulting the culture and sensitivity testing. Outcome was documented in terms of mortality, morbidity, and hospital stay. Outcome parameters were correlated with factors including delay in presentation, co-morbid conditions, bacterial culture, antibiotic use and changes.

The Chi-square test or Fisher's exact test was used for comparing proportions. Prognostic factors were determined as per the demographic data, patient's history, severity of the illness, surgical management, microbiological data and patient's outcome. Univariate analysis of prognostic factors was performed using Student's t-test or the Kruskal-Wallis test for comparing quantitative parameters. A multivariate analysis was carried out using a stepwise logistic regression to control all confounding factors and find the independent predictors of mortality.

\section{Results}

We enrolled 352 patients of secondary bacterial peritonitis in this study (Table 1). The male:female ratio was 7:1. The agewise distribution of patients is presented in Table 2 . The median age of the patients was 44 years (range: 13-85 yrs). Gastroduodenal (51\%) and small bowel perforations (29\%) were the common causes of peritonitis. Majority (75\%) of the patients presented to us one to three days after the onset of symptoms. Only 37 (11\%) patients presented within 24 hours of the onset and 10 patients (3\%) presented with symptoms beyond 4 days. Pain abdomen was a universal presenting symptom, followed by abdominal distension (71\%) and vomiting (42\%). Fever was present in $123(34 \%)$ patients and $64(18 \%)$ patients gave history of taking over-the-counter analgesics. Generalized abdominal tenderness was present in 295 (83\%) patients while 65 (18\%) 
Table 1: Etiology and gender distribution

\begin{tabular}{lllll}
\hline Site of perforation & Males & Females & Total & $(\boldsymbol{\%})$ \\
\hline $\begin{array}{l}\text { Gastroduodenal }(\mathrm{n}=180) \\
\text { - Duodenal ulcer }\end{array}$ & 138 & 8 & 146 & $(41.4)$ \\
- Gastric ulcer & 26 & 4 & 30 & $(8.5)$ \\
- Carcinoma stomach & 4 & - & 4 & $(1.1)$ \\
Small bowel (n=102) & & & & \\
- Non-specific & 46 & 8 & 54 & $(15.0)$ \\
- Traumatic & 14 & - & 14 & $(4.0)$ \\
- Typhoid & 10 & 2 & 12 & $(3.4)$ \\
- Ischemic & 7 & 2 & 9 & $(2.5)$ \\
- Radiation enteritis & - & 8 & 8 & $(2.2)$ \\
- Tubercular & 4 & 1 & 5 & $(2.0)$ \\
Colorectal (n=6) & & & & \\
- Carcinoma colon & 1 & 1 & 2 & $(0.5)$ \\
- Non-specific & 1 & 1 & 2 & $(0.6)$ \\
- Traumatic & 1 & - & 1 & $(0.3)$ \\
- Radiation proctitis & - & 1 & 1 & $(0.3)$ \\
Appendicular (n=59) & 51 & 8 & 59 & $(16.7)$ \\
Gall bladder (n=5) & 4 & 1 & 5 & $(1.4)$ \\
\hline Total $(\%)$ & $\mathbf{3 0 7 ( 8 7 . 0 )}$ & $\mathbf{4 5 ( 1 3 . 0 )}$ & $\mathbf{3 5 2}$ & $(\mathbf{1 0 0 . 0 )}$ \\
\hline
\end{tabular}

patients presented with shock. Chest X-ray showed pneumoperitoneum in 250 patients which was noted in $94 \%$ of gastroduodenal perforations, $68 \%$ of small bowel perforations and in only $7 \%$ of appendicular perforations. $28.5 \%$ of patients had organ dysfunction at presentation. The most common being renal dysfunction (9\%), followed by cardiovascular (4.2\%) and pulmonary (3.6\%) dysfunction.

Peritoneal lavage, omental patch closure, with or without feeding jejunostomy formed the mainstay of treatment of gastroduodenal perforations. Resection and anastomosis was the most common surgery done for small bowel perforations. Appendicectomy, lavage and drainage was done for appendicular perforation. Colorectal perforations were treated with resection, lavage and stoma. There was clinical evidence of failure of source control in 51 (14.5\%) patients, of which 38 (10.8\%) underwent repeat laparotomy. Rest of the patients were either conservatively managed as per surgeon's discretion or were too sick to be taken up for repeat laparotomy.

A total of 227 (64\%) patients had positive peritoneal fluid culture. Escherichia coli (35\%) was the dominant isolate in all perforation sites, followed by Klebsiella, Streptococcus and Acinetobacter species. Candida alone was isolated in 13 cases. No anaerobes were isolated in the 18 cases of lower gastrointestinal perforation tested for them (Table 3). Three patients were found infected with three organisms while 18 patients had two organism infections. Incidence of culture positivity was $76 \%$ in non-survivors compared to $59 \%$ in survivors. Isolation of Enterococcus species or the presence of multiple isolates was not associated with adverse outcome by univariate analysis. In vitro susceptibility testing of these isolates showed that $E$. coli isolates were mostly sensitive to amikacin (94\%) followed by ceftazidime (91\%) and chloramphenicol (91\%). Klebsiella species were sensitive to cephalosporins, aminoglycosides and ciprofloxacin. (Table 4)

Ampicillin with gentamicin and metronidazole were the first line agents used preoperatively in 234 (67\%) patients.

Table 2: Age distribution

\begin{tabular}{lcccccc}
\hline Age in years & $\begin{array}{c}\text { Gastroduodenal } \\
(\mathbf{n = 1 8 0})\end{array}$ & $\begin{array}{c}\text { Small bowel } \\
\mathbf{n = 1 0 2}\end{array}$ & $\begin{array}{c}\text { Appendicular } \\
\mathbf{n = 5 9}\end{array}$ & $\begin{array}{c}\text { Colorectal } \\
\mathbf{n = 6}\end{array}$ & $\begin{array}{c}\text { Gall bladder } \\
\mathbf{n = 5}\end{array}$ & $\begin{array}{c}\text { Total } \\
(\mathbf{\%})\end{array}$ \\
\hline$<20$ & 2 & 6 & 2 & - & - & $10(2.8 \%)$ \\
$20-29$ & 24 & 12 & 10 & - & - & $46(13.1 \%)$ \\
$30-39$ & 47 & 27 & 12 & - & 1 & $87(24.7 \%)$ \\
$40-49$ & 58 & 32 & 20 & 1 & 2 & $73(20.7 \%)$ \\
$50-59$ & 29 & 20 & 12 & 2 & 2 & $75(21.3 \%)$ \\
$>60$ & 20 & 5 & 3 & 3 & $61(17.3 \%)$ \\
\hline
\end{tabular}

Table 3: Culture results of peritoneal fluid at primary surgery.

\begin{tabular}{|c|c|c|c|c|c|}
\hline Organisms & $\begin{array}{c}\text { Gastro duodenal } \\
(n=180)\end{array}$ & $\begin{array}{c}\text { Small bowel } \\
(n=102)\end{array}$ & $\begin{array}{c}\text { Appendicular } \\
(\mathrm{n}=\mathbf{5 9})\end{array}$ & $\begin{array}{c}\text { Colo rectal } \\
(n=6)\end{array}$ & $\begin{array}{c}\text { Gall bladder } \\
(n=5)\end{array}$ \\
\hline$\overline{\text { Escherichia coli }}$ & 33 & 22 & 20 & 4 & 2 \\
\hline Klebsiella sp. & 23 & 11 & 8 & 1 & - \\
\hline Enterococcus sp. & 9 & 10 & 6 & - & - \\
\hline Proteus sp. 5 & 7 & 6 & 2 & - & \\
\hline Streptococcus sp. & 15 & 5 & 1 & - & - \\
\hline Acinetobacter sp. & 5 & 5 & 3 & - & - \\
\hline Candida sp. & 9 & 4 & - & - & - \\
\hline Others & 9 & 6 & 4 & - & - \\
\hline Total isolates (positive culture) & $108(100)$ & $70(64)$ & $48(42)$ & $7(5)$ & $2(2)$ \\
\hline Culture negative $(\%)$ & $80(45)$ & $38(38)$ & $17(29)$ & $1(17)$ & $3(60)$ \\
\hline
\end{tabular}


Table 4: Antibiotic sensitivity profile of the common peritoneal fluid isolates.

\begin{tabular}{|c|c|c|c|c|c|c|c|}
\hline Organisms & $\begin{array}{c}A \\
(\%)\end{array}$ & $\begin{array}{c}\mathbf{G} \\
(\%)\end{array}$ & $\begin{array}{l}\mathbf{A k} \\
(\%)\end{array}$ & $\begin{array}{c}\mathbf{C i} \\
(\%)\end{array}$ & $\begin{array}{l}\mathrm{Ca} \\
(\%)\end{array}$ & $\begin{array}{l}\mathrm{Va} \\
(\%)\end{array}$ & $\begin{array}{l}\mathbf{C f} \\
(\%)\end{array}$ \\
\hline Escherichia colin $=81$ & 12 & 17 & 94 & 36 & 91 & - & 17 \\
\hline Klebsiellan $=43$ & 54 & 73 & 76 & 92 & 86 & - & 73 \\
\hline Enterococcusn $=25$ & 76 & 88 & - & - & - & 92 & - \\
\hline Streptococcusn $=21$ & 70 & 90 & - & - & - & 95 & - \\
\hline Acinetobactern $=13$ & 77 & 85 & 92 & 92 & 92 & - & 85 \\
\hline
\end{tabular}

A-Ampicillin; G-Gentamicin; Ak-Amikacin; Ci-Ceftriaxone; Ca-Ceftazidime;

$V$-Vancomycin; $C f$ - Ciprofloxacin

Table 5: Antibiotic regimes instituted in 352 patients

\begin{tabular}{|c|c|c|c|c|c|}
\hline \multirow[t]{2}{*}{ Antibiotic combinations used } & \multirow{2}{*}{$\begin{array}{l}\text { Number of } \\
\text { patients who } \\
\text { received } \\
\text { preoperatively }\end{array}$} & \multirow[b]{2}{*}{$\begin{array}{l}\text { Number of } \\
\text { patients who } \\
\text { received in the } \\
\text { immediate } \\
\text { postoperative } \\
\text { period }\end{array}$} & \multicolumn{2}{|c|}{ Postoperative course } & \multirow[b]{2}{*}{$\begin{array}{c}\text { Number of } \\
\text { patients who } \\
\text { additional course } \\
\text { for infective } \\
\text { causes }\end{array}$} \\
\hline & & & $\begin{array}{l}\text { Number of } \\
\text { patients who } \\
\text { received } \\
\text { to use }\end{array}$ & $\begin{array}{c}\text { Number of } \\
\text { patients who } \\
\text { continued were } \\
\text { changed to }\end{array}$ & \\
\hline Ampicillin + Gentamicin + Metronidazole & 234 & 155 & 112 & - & - \\
\hline Ceftriaxone + Amikacin + Metronidazole & 40 & 102 & 92 & 14 & - \\
\hline Ceftriaxone + Gentamicin + Metronidazole & 25 & 34 & 26 & 6 & - \\
\hline Cephalosporin + Metronidazole & 39 & 37 & 18 & - & - \\
\hline Ceftazidime + Amikacin + Metronidazole & 6 & 16 & 11 & 17 & 8 \\
\hline Meropenem & - & - & - & 12 & 24 \\
\hline Cefoperazone + Sulbactum & - & - & - & 14 & 12 \\
\hline Vancomycin & - & - & - & 4 & 14 \\
\hline Ciprofloxacin + Metronidazole & 8 & 8 & 6 & 4 & 12 \\
\hline Other antibiotics & - & - & - & - & 21 \\
\hline
\end{tabular}

Table 6: Antibiotic usage with reference to culture antibiogram in $227^{\dagger}$ patients

\begin{tabular}{lc}
\hline Preoperative antibiotics & $\mathbf{n}^{\dagger}$ \\
\hline - Nodequate $(\mathrm{n}=170)$ & \\
- Appropriate change & 93 \\
- Inappropriate change & 50 \\
Adequate ( $\mathrm{n}=47)$ & 23 \\
- No change or inappropriate change & \\
- Appropriate change & 38 \\
\hline$\dagger$ Numbers do not add up due to isolation of Candida alone in 10 \\
patients or lack of sensitivity data on cefoperazone and meropenem \\
in few cases.
\end{tabular}

Cephalosporin with aminoglycoside and metronidazole formed the preferred second line combination for empiric changes and postoperative changes following culture results (Table 5). 78\% of patients received inadequate antibiotics preoperatively. Only $26 \%$ had appropriate change of antibiotics. However there was no significant difference in hospital stay, repeat laparotomy rate, morbidity or mortality between these groups. (Tables $6 \mathbf{\&}$ 7)

APACHE II (Acute Physiology and Chronic Health Evaluation) score at admission was available for 114 patients of whom 32 expired. The median APACHE II score in survivors was 7 versus 17 in non-survivors. Incidence of overall

Table 7: Effect of postoperative changes in antimicrobial therapy in patients with positive culture

\begin{tabular}{|c|c|c|c|c|c|c|}
\hline Postoperative antibiotics & $\begin{array}{c}\mathbf{n}^{\dagger} \\
\text { stay (days) }\end{array}$ & $\begin{array}{c}\text { Mean hospital } \\
\text { operation }\end{array}$ & $\begin{array}{c}\text { Re- } \\
\text { infection }\end{array}$ & $\begin{array}{c}\text { Wound } \\
\text { morbidity }\end{array}$ & Total & Deaths $^{\dagger}$ \\
\hline \multicolumn{7}{|l|}{ Inadequate $(\mathrm{n}=170)$} \\
\hline - No change or inappropriate & 116 & 9.5 & 11 & 56 & 64 & 17 \\
\hline - Appropriate change & 50 & 10.7 & 5 & 16 & 18 & 13 \\
\hline \multicolumn{7}{|l|}{ Adequate $(n=47)$} \\
\hline - No change or inappropriate & 38 & 10.4 & 3 & 20 & 23 & 10 \\
\hline - Appropriate change & 4 & 11.2 & 2 & 3 & 4 & 1 \\
\hline$\underline{P}$ value & & 0.41 & 0.28 & 0.50 & 0.07 & 0.12 \\
\hline
\end{tabular}

$\uparrow$ Numbers do not add up due to isolation of Candida alone in 10 patients and lack of sensitivity data on cefoperazone and meropenem in 9 cases including 3 expired patients. 
morbidity was $89 \%$ in non-survivors as compared to $44 \%$ in survivors $(\mathrm{p}<0.001)$. The mean total ICU (Intensive Care Unit) stay in non-survivors was $5.6 \pm 3.4$ days compared to $2.4 \pm 1.6$ days in survivors $(\mathrm{p}<0.001)$. The mortality rate was $16.5 \%$. By univariate analysis, increasing age, female sex, delay in presentation, preoperative organ dysfunction, high APACHE II score, postoperative hemoglobin level, non-appendicular origin of peritonitis, positive culture, failure of source control at surgery, prolonged ICU stay and presence of postoperative morbidity were found to be significantly associated with risk of mortality (Table 8). Multivariate analysis using binary logistic regression test revealed only age $>60$ years, delay $>3$ days, and APACHE II score $>15$ were found to be independent predictors of mortality.

Table 8: Factors associated with mortality

\begin{tabular}{|c|c|c|c|}
\hline \multirow[t]{2}{*}{ Parameters } & \multicolumn{3}{|c|}{ Survivors Non-survivors $p$ value } \\
\hline & $(n=294)$ & $(\mathbf{n}=\mathbf{5 8})$ & \\
\hline$\overline{\text { Median age (SD) }}$ & $40(9.6)$ & $55(10.4)$ & 0.001 \\
\hline Female $(\%)$ & $28(9.5)$ & $17(29)$ & 0.002 \\
\hline $\begin{array}{l}\text { Mean delay in } \\
\text { presentation in days (SD) }\end{array}$ & $2.4(1.9)$ & $3.8(1.6)$ & 0.002 \\
\hline $\begin{array}{l}\text { Mean delay in surgery } \\
\text { in hours (SD) }\end{array}$ & $7.4(3.3)$ & $8.6(4.7)$ & 0.067 \\
\hline $\begin{array}{l}\text { Preoperative organ } \\
\text { impairment }(\%)\end{array}$ & $42(14.2)$ & $23(39)$ & 0.001 \\
\hline Mean hemoglobin (g/dl) & $10.8(4.3)$ & $8.6(4.1)$ & 0.016 \\
\hline Culture positivity (\%) & $173(59)$ & $44(76)$ & 0.018 \\
\hline Median APACHE II score & 7 & 17 & $<0.0001$ \\
\hline Repeat laparotomy (\%) & $29(10)$ & $9(14)$ & 0.24 \\
\hline Failure at source control (\%) & $24(8)$ & $27(47)$ & $<0.0001$ \\
\hline Postop morbidity (\%) & $132(44)$ & $52(89)$ & 0.001 \\
\hline Mean ICU stay in days (SD) & $2.4(1.6)$ & $5.6(3.4)$ & $<0.0001$ \\
\hline Mean hospital stay in days (SD) & $9.8(6.4)$ & $12.6(7.2)$ & 0.06 \\
\hline
\end{tabular}

\section{Discussion}

This study aimed at studying the factors affecting outcome of community acquired acute bacterial peritonitis with special focus on routine peritoneal fluid culture and its relevance in antibiotic therapy. Secondary bacterial peritonitis due to gastrointestinal perforation is a common surgical emergency at tertiary care centers like JIPMER in India. Gastroduodenal perforations $(51 \%)$ due to peptic ulcers were the major cause of peritonitis in our study similar to most studies from the eastern hemisphere..$^{4-8}$ However, Agarwal et $\mathrm{al}^{9}$ and Dorarajan et $\mathrm{al}^{10}$ found small bowel perforations more common in North India because of decreasing incidence of peptic ulcer. In the West and in developed countries, it is well known that large bowel perforations are more common. ${ }^{11-13}$ In our study, nonspecific perforations accounted for $52 \%$ of small bowel perforations with typhoid related perforations being reported in lesser numbers, compared to earlier eastern literature. ${ }^{7,8,10}$ Non-specific perforations are postulated to be secondary to submucous vascular embolism ${ }^{14}$ or ischemia due to atheromatous arterial disease and arteritis. ${ }^{15}$ Etiopathogenesis of this entity warrants further study. Peritonitis due to radiation enteritis was exclusively seen in eight patients treated for carcinoma cervix. Appendicular and colorectal perforation was the etiology in $19 \%$ of our cases, similar to that noted by $\mathrm{Khan}^{4}$ and Dorairajan. ${ }^{10}$

In our study $47 \%$ patients presented after 48 hours of onset of acute symptoms. This is similar to that noted in previous studies from our institute, ${ }^{16,17}$ where $37 \%$ of the study population presented beyond 24 hours. After a few days, peritonitis is no longer a localized problem and limits the surgeon's ability to securely eliminate the focus of infection. ${ }^{18,19}$ The mean delay in taking up surgery from the time of diagnosis was higher but not significantly so in non-survivors (8.6 vs. 7.4 hours), an observation which differs from published literature. ${ }^{18,19} 28.5 \%$ of patients had preoperative organ dysfunction. Preoperative organ dysfunction or concomitant medical illness has been shown to be associated with increased mortality. ${ }^{19-22}$ There was relatively higher incidence of renal failure in our study which is different from other series where cardiovascular, pulmonary and malignant conditions comprised of the major co-morbid conditions. Our study population was considered homogenous with regards to surgical treatment, with standard source control surgeries with peritoneal lavage with saline solution and drains based on the degree of peritoneal contamination. 51 patients experienced a failure at source control, of which 38 (10.8\%) underwent repeat laparotomy. Rest of the patients were managed either conservatively as per attending surgeon's discretion or were too sick to be taken up for repeat laparotomy. $47 \%$ of non-survivors failed at source control as compared to $8 \%$ survivors $(\mathrm{p}<0.0001)$.

The culture positivity rate of $64 \%$ in our study and was lower than other series. ${ }^{23-27}$ However, the spectrum of isolates matched with other series with E. coli and Klebsiella being the dominant isolates. Only $22 \%$ received adequate preoperative antibiotics but the mean hospital stay, wound infection rates, overall morbidity rates and mortality rates were the same when compared with those receiving inadequate antibiotics. Only $26 \%$ had appropriate postoperative change of antibiotics, but this had no effect on morbidity or mortality. The absence of correlation between adapted antibiotic therapy and expected outcome in our study has been frequently 
reported in literature. ${ }^{1,3,23,28}$ The most important reason could be the delay in presentation. Instituting appropriate antibiotics at later stages may be ineffective..$^{29,30}$

Analysis of 114 patients, revealed a median APACHE II score of 7 in survivors was 7 and 17 in non-survivors. The difference was statistically significant and the same has been reported earlier. ${ }^{31,32}$ The pattern of various complications was consistent with the spectrum of morbidity reported in literature ${ }^{7,12,13,33-5}$ except for the higher rate of wound infection. The mortality rate in our series was $16.5 \%$. Other studies have reported $9 \%$ to $26 \%$ mortality, approaching $30 \%$ in severe intraabdominal infection. ${ }^{33-36}$ The higher percentage of females in the non-survivors group compared to total study population was associated with radiation enteritis which occurred exclusively in females who received radiotherapy for carcinoma of cervix. By multivariate analysis only age $>60$ years, delay $>3$ days and APACHE II score $>15$ were independently and significantly associated with increased risk of mortality. This shows that host related factors have a much greater impact on survival in patients with peritonitis. ${ }^{20,21}$ Some of the limitations of our study include non-availability of APACHE II scores for all patients and institution of higher antibiotics as per availability.

Unlike in North India, in our study of community acquired acute secondary bacterial peritonitis, gastroduodenal perforations comprised the major site of perforation (51\%), followed by small bowel (29\%) and appendicular perforations $(17 \%)$. The spectrum of bacterial isolates from peritoneal fluid was consistent with previous reports, with Escherichia coli and Klebsiella as most common isolates. The mortality and morbidity rates are comparable in spite of lack of correlation with in-vitro bacteriological susceptibility reports in evaluable patients. Since the data is equivocal about potential benefit of postoperative change of antibiotics according to culture results, it may be prudent to adapt antibiotics as per culture results in patients who are critically ill or those unresponsive to empiric therapy. Analysis of factors influencing mortality shows dominance of host related factors over the type and source of infection with high risk population identified by age $>60$ years, delayed presentations $>3$ days and APACHE II score $>15$.

\section{References}

1. Mosdell DM, Morris DM, Voltura A, Pitcher DE, Twiest MW, Milne RL, et al. Antibiotic treatment for surgical peritonitis. Ann
Surg. 1991;214:543-9.

2. Nathens AB. Relevance and utility of peritoneal cultures in patients with peritonitis. Surg Infect (Larchmt). 2001;2:153-60; discussion 60-2.

3. Wittmann DH, Schein M and Condon RE. Management of secondary peritonitis. Ann Surg. 1996;224:10-8.

4. Khan S, Khan IU, Aslam S and Haque A. Retrospective analysis of abdominal surgeries at Nepalgunj Medical College (NGMC), 2004;2:336-43.

5. Nishida T, Fujita N, Megawa T, Nakahara M and Nakao K. Postoperative hyperbilirubinemia after surgery for gastrointestinal perforation. Surg Today. 2002;32:679-84.

6. Chen SC, Lin FY, Hsieh YS and Chen WJ. Accuracy of ultrasonography in the diagnosis of peritonitis compared with the clinical impression of the surgeon. Arch Surg. 2000;135:1703; discussion 4.

7. Gupta S and Kaushik R. Peritonitis - the Eastern experience. World J Emerg Surg. 2006;1:13.

8. Sharma L, Gupta S, Soin AS, Sikora S and Kapoor V. Generalized peritonitis in India-the tropical spectrum. Jpn J Surg. 1991;21:272-7.

9. Agarwal N, Saha S, Srivastava A, Chumber S, Dhar A and Garg S. Peritonitis: 10 years' experience in a single surgical unit. Trop Gastroenterol. 2007;28:117-20.

10. Dorairajan LN, Gupta S, Deo SV, Chumber S and Sharma L. Peritonitis in India - a decade's experience. Trop Gastroenterol. 1995;16:33-8.

11. Crawford E and Ellis H. Generalised peritonitis-the changing spectrum. A report of 100 consecutive cases. Br J Clin Pract. 1985;39:177-8, 84.

12. Malangoni MA and Inui T. Peritonitis - the Western experience. World J Emerg Surg. 2006;1:25.

13. Mulari K and Leppaniemi A. Severe secondary peritonitis following gastrointestinal tract perforation. Scand J Surg. 2004;93:204-8.

14. Evert JA, Black BM and Dockerty MB. Primary nonspecific ulcers of the small intestine. Surgery. 1948;23:185-200.

15. Finkbiner RB. Ulceration and perforation of the intestine due to necrotizing arteriolitis. N Engl J Med. 1963;268:14-7

16. Krishna KG, Pai D. A study of factors contributing to releak after surgical closure of perforated of perforated duodenal ulcer. Dissertation submitted to Pondicherry University. 2000.

17. Rajesh V, Chandra SS and Smile SR. Risk factors predicting operative mortality in perforated peptic ulcer disease. Trop Gastroenterol. 2003;24:148-50.

18. Svanes C, Lie RT, Svanes K, Lie SA and Soreide O. Adverse effects of delayed treatment for perforated peptic ulcer. Ann Surg. 1994;220:168-75.

19. Sharma SS, Mamtani MR, Sharma MS and Kulkarni H. A prospective cohort study of postoperative complications in the management of perforated peptic ulcer. BMC Surg. 2006;6:8.

20. Pacelli F, Doglietto GB, Alfieri S, Piccioni E, Sgadari A, Gui D, et al. Prognosis in intra-abdominal infections. Multivariate analysis on 604 patients. Arch Surg. 1996;131:641-5.

21. Wacha H, Hau T, Dittmer R and Ohmann C. Risk factors associated with intraabdominal infections: a prospective multicenter study. Peritonitis Study Group. Langenbecks Arch 
Surg. 1999;384:24-32.

22. Wakayama T, Ishizaki Y, Mitsusada M, Takahashi S, Wada T, Fukushima Y, et al. Risk factors influencing the short-term results of gastroduodenal perforation. Surg Today. 1994;24:681-7.

23. Sotto A, Lefrant JY, Fabbro-Peray P, Muller L, Tafuri J, Navarro F, et al. Evaluation of antimicrobial therapy management of 120 consecutive patients with secondary peritonitis. J Antimicrob Chemother. 2002;50:569-76.

24. Christou NV, Turgeon P, Wassef R, Rotstein O, Bohnen J and Potvin M. Management of intra-abdominal infections. The case for intraoperative cultures and comprehensive broad-spectrum antibiotic coverage. The Canadian Intra-abdominal Infection Study Group. Arch Surg. 1996;131:1193-201.

25. Budhiraja SN, Chidambaram M, Perianayagam WJ. Peritonitis an analyasis of 117 cases. Ind J Surg. 1983;35:456-64.

26. Santos SG, Serufo JC, Silva RA, Marra BA, Reis CM, Hamdan JS, et al. Microbiologic profile of intra-abdominal infections at Belo Horizonte, Brazil. Am J Infect Control. 2003;31:135-43.

27. Hopkins JA, Lee JC and Wilson SE. Susceptibility of intraabdominal isolates at operation: a predictor of postoperative infection. Am Surg. 1993;59:791-6.

28. Mosdell DM, Morris DM and Fry DE. Peritoneal cultures and antibiotic therapy in pediatric perforated appendicitis. Am J Surg. 1994;167:313-6.

29. Farthmann EH and Schoffel U. Epidemiology and pathophysiology of intraabdominal infections (IAI). Infection. 1998;26:329-34.

30. Christou NV. Host defense mechanisms of surgical patients. Friend or foe? Arch Surg. 1996;131:1136-40.

31. Naveen T, Chandra SS, Smile SR. Use of APACHE II scoring in assessing patients with generalized peritonitis. Dissertation submitted to Pondicherry University 1998.

32. Kumar PS, Rao SC. Prognosis in intraabdominal sepsis. Indian J Gastroenterol. 1995;14:8-10.

33. Jhobta RS, Attri AK, Kaushik R, Sharma R and Jhobta A. Spectrum of perforation peritonitis in India-review of 504 consecutive cases. World J Emerg Surg. 2006;1:26.

34. Afridi SP, Malik F, Ur-Rahman S, Shamim S and Samo KA. Spectrum of perforation peritonitis in Pakistan: 300 cases Eastern experience. World J Emerg Surg. 2008;3:31.

35. Gupta SK, Gupta R, Singh G, Gupta S. Perforation peritonitis: A two year experience. JK Science. 2010;12:141-4.

36. Schein M. Management of severe intra-abdominal infection. Surg Апnи. 1992;24 :47-68. 